\title{
Application Prospect of Artificial Intelligence in Rehabilitation and Management of Myasthenia Gravis
}

\author{
Ying Zhang $\mathbb{D}^{1}{ }^{1}$ Hongmei Yu, ${ }^{1}$ Rui Dong, ${ }^{1}$ Xuan Ji, ${ }^{1}$ and Fujun $\mathrm{Li}\left(\mathbb{D}{ }^{2}\right.$ \\ ${ }^{1}$ Department of Neurology, The Second Affiliated Hospital of Harbin Medical University, Harbin, Heilongjiang, China \\ ${ }^{2}$ Department of General Surgery, The Second Affiliated Hospital of Harbin Medical University, Harbin, Heilongjiang, China
}

Correspondence should be addressed to Fujun Li; 55977@163.com

Received 13 January 2021; Revised 20 February 2021; Accepted 24 February 2021; Published 4 March 2021

Academic Editor: Lei Jiang

Copyright (c) 2021 Ying Zhang et al. This is an open access article distributed under the Creative Commons Attribution License, which permits unrestricted use, distribution, and reproduction in any medium, provided the original work is properly cited.

\begin{abstract}
Myasthenia gravis (MG) is a chronic autoimmune disease of the nervous system, which is still incurable. In recent years, with the progress of immunosuppressive and supportive treatment, the therapeutic effect of MG in the acute stage is satisfactory, and the mortality rate has been greatly reduced. However, there is still no consensus on how to conduct long-term management of stable MG, such as guiding patients to identify relapses, practice exercise, return to work and school, etc. In the international consensus guidance for management of myasthenia gravis published by the Myasthenia Gravis Foundation of America (MGFA) in 2020, for the first time, "the role of physical training/exercise in MG" was identified as the topic of discussion. Finally, due to a lack of high-quality evidence on physical training/exercise in patients with MG, the topic was excluded after the literature review. Therefore, this paper reviewed the current status of MG rehabilitation research and the difficulties faced by stable MG patients in self-management. It is suggested that we should take advantage of artificial intelligence (AI) and leverage it to develop the data-driven decision support platforms for MG management which can be used for adverse event monitoring, disease education, chronic management, and a wide variety of data collection and analysis.
\end{abstract}

\section{Introduction}

Myasthenia gravis (MG) is a chronic autoimmune disease affecting the postsynaptic membrane at the neuromuscular junction thereby obstructing nerve impulse transmission $[1$, 2]. The prevalence rate (PR) of MG is 15 to 179 cases per million, estimated pooled PR is 77.7; and the incidence rate (IR) is 1.7 to 21.3 cases per million person-years, estimated pooled IR is 5.3 [3]. MG treatment traditionally includes the symptomatic treatment by acetylcholinesterase inhibitors, thymectomy, steroids and/or nonsteroidal immunosuppressive therapy, intravenous immunoglobulin (IVIg), or plasmapheresis. Advancements in therapeutics have significantly reduced MG-associated mortality $[4,5]$. However, the disease still affects most patients' daily functional activities and reduces their quality of life [6-9]. Since it is difficult for patients with MG to achieve complete stable response (CSR), the current international consensus has proposed minimum performance state (MMS) or better as the treatment goal of MG [10]. Therefore, the chronic management of MG includes complex pharmacotherapy and lifestylerelated activities, which must be optimized to improve the quality of life in patients and enable as many patients as possible to achieve MMS or better treatment goals. In the past few years, more and more AI-based software and hardware have been developed for the management and clinical research of chronic diseases. In this review, we introduce the successful experience of AI in several chronic disease management and discuss the possible application of $\mathrm{AI}$ in the rehabilitation and management of $M G$ in the future.

\section{Current Status and Dilemma of Rehabilitation and Management in MG}

For a long time, physicians have paid more attention to the treatment of acute symptoms in MG; however, the potentially prolonged course of the disease remains neglected. In many MG patients, even if symptoms are improved by standardized treatment after onset, they will soon be plagued again by disease recurrence or drug-related adverse events. 
Here are several common reasons: (1) relapse as a consequence of early withdrawal of medication, undertaken due to resolution of symptoms; (2) adverse events caused by failure to regularly monitor the safety of medication; (3) other chronic diseases caused by long-term use of glucocorticoids and immunosuppressive drugs; and (4) relapse of MG for unknown causes. With the prolongation and fluctuation of the disease, MG patients are often puzzled over the period of their treatment. "How much and how long should I take my medicine? What should I pay attention to in my daily life? Can I do physical exercise? When can I go to work? Why is my sleep getting worse?" Even in the recently published consensus guidance for management [11], the expert group did not give clear recommendations for the management of stable MG patients. Some studies have shown that most MG patients are often restricted when engaging in social activities, such as entertainment or work. Patients present with depression, anxiety, social reclusiveness, and frustration to a certain extent, arising from their MG [12-14]. Therefore, treatment strategies should also include the long-term management of MG and aim towards an improved quality of life and mental health [15].

The preventive benefits of physical exercise in various chronic diseases such as stroke, heart diseases, and cancer are well known, and its role in promoting positive psychological effects is beginning to be widely accepted at the same time [16-18]. Physical exercise has even been compared to drug therapy and has been therefore recommended as part of the management of many chronic diseases.

Traditionally, it was believed that physical training/exercise exacerbated symptoms for MG patients. However, the current general opinion supports that MG patients can benefit from physical exercise, which the evidences coming from a small number of physical exercise clinical trials. In a stratified study by Rahbek et al. [19], patients were assigned randomly to either the aerobic training (AT) group or the progressive resistance training (RT) group intervention over 8 weeks. Primary results showed that MG patients were well tolerated to both types of physical intervention within a specified time period (i.e., 8 weeks). Secondary results showed that muscle strength and functional capacity improved in the RT group, compared with no change in the AT group. A prospective study, by Westerberg et al. [20], supervised AT and RT twice weekly for 12 weeks with MG patients. While the study did not report increased muscle strength but the patient performed significantly better on the physical fitness tests such as the 6-min walk test and 30-second-sit-tostand test. Another study incorporating respiratory training on stable MG patients for a period of 8 weeks used a casecontrol approach by randomly dividing patients into cohorts. The patients were trained for diaphragmatic breathing and pursed lips breathing. Compared to the controls and to the individual baseline values, patients had improved respiratory muscle endurance, maximum inspiratory and expiratory pressures, and thoracic mobility [21].

In the international consensus guidance for management of myasthenia gravis: 2020 update [11], for the first time, "the role of physical training/exercise in $M G$ " was identified as the topic of discussion, which shows that more and more experts have begun to pay attention to the exercise rehabilitation and chronic management of MG patients. However, the low quality of evidence with respect to physical training/exercise and its significance in the management of chronic MG led to its noninclusion as an informing recommendation. So, what are the factors that limit the design and completion of highquality clinical trials on physical exercise in MG? We summarized the following reasons: (1) it is difficult to recruit subjects. The first step in MG clinical trial is to select subjects based on strict and well-defined criteria for inclusion and exclusion, such as age, course of disease, subtype, severity, concomitant diseases, and received interventions. In addition, the low incidence rate of MG further increases the difficulty of recruitment. Each trial will be recruited within a certain time window. Enrolling enough patients within the specified time is a necessary condition for the success of a trial; (2) in order to ensure the real and effective exercise data, trainings need supervision, which limit the activity scope and activity time of subjects and reduce their compliance; (3) prolonged interventions will introduce more influence factors, making prospective study designs difficult; (4) selection criteria for control group is unclear; (5) double-blind trials cannot be designed; (6) absence of clarity or consensus with respect to standard outcomes in MG is missing.

\section{Application of AI in the Management of Chronic Diseases}

3.1. Artificial Intelligence Techniques. The basic idea of artificial intelligence (AI) is to simulate human thinking through a computer, that is, the process of perceiving the world, learning constantly, and making decisions. AI is an emerging field in computer sciences which involve human independent application of specifically designed computer algorithms. AI approaches fall into three categories [22]: (1) exploration and discovery of knowledge, this is also known as knowledge discovery in databases (KDD). KDD is primarily used for identifying information validity. It segregates information as relevant/useful and nonrelevant/nonuseful. One of the important steps towards knowledge discovery is data mining. Data mining technology mainly includes decision tree, neural network, regression, association rule, clustering, and Bayesian classifier; (2) learning from knowledge, the method permits learning from the acquired knowledge. Computers can learn on their own without any assistance or intervention from humans. The method is aimed to enable better decision-making and more accurate predictions about future conditions; (3) reasoning from knowledge is the third method. In this method, the existing knowledge becomes the starting point, and using logical techniques, such as deduction and induction, a hypothesis is proved or disproved and helps derive conclusions. For instance, an intelligent medical diagnosis system can diagnose based on the clinical and pathological presentation of the disease, using the knowledge in the databases and the control strategies.

3.2. Emerging Application of AI in Medicine. AI was first used in medicine in the 1970s, but the development in the field of medicine has been slow in the following decades. Until the 
last few years, with the development of information and communication technologies, things have changed. First, the rapid development of software and hardware technology makes it possible to obtain widely available medical data. The popularity of electronic health records (EHR) and the emergence of sensors and wearable devices are promoting the evolution of medical data to digital form. The massive medical data obtained can be processed in real time and efficiently by using big data analysis technology, such as machine learning, deep learning, and data mining. Secondly, the real-time transmission and sharing of information are realized through the Internet. $5 \mathrm{G}$ communication technology is about to promote the transformation of mobile Internet to the Internet of everything. The progress and combination of these technologies are driving new developments in the field of medical technology. At present, AI is widely used in health technology. They are utilized at every stage of disease management, including disease screening, [23, 24], disease diagnosis [25-28], prognosis estimation [29, 30], decision support [31], and therapeutic recommendation [32]. Recently, AI attracted considerable interest for its advantages in health and chronic disease management $[33,34]$.

3.3. Examples of AI Applications in Chronic Disease Management. The full name of chronic diseases is chronic noncommunicable diseases (NCDs). The common chronic diseases mainly include cancer, diabetes, hypertension, obesity, chronic respiratory diseases, chronic kidney diseases, autoimmune diseases, cardiovascular and cerebrovascular diseases, and neurodegenerative diseases [35]. Globally, chronic diseases are known to affect nearly a quarter of the adult population and are known to have a huge negative socioeconomic impact [34]. In the following, we will introduce some examples of AI application in chronic disease management and related research.

Chronic disease management requires patient monitoring advice and status assessment. This is one of the application areas to explore AI methods in combination with other mobile computing and sensor technologies, which is expected to create and provide better services for chronic disease management. For instance, in diabetes, AI methods are used for blood sugar monitoring, lifestyle recommendations, and self-management. Exploration of a computerized decision support system (DSS) for diabetes has been undertaken. These computer applications monitor disease outcomes by recording information about diet, exercise, drug use, and blood glucose levels $[36,37]$. The utility of such digital technology can also be seen in the management of chronic lung conditions. Res-App is used for monitoring patient breathing through the phone microphone providing an evaluation for several lung diseases, such as asthma, pneumonia, lower respiratory tract disease, croup, and bronchiolitis [38]. Altogether, a combination of sensor-based and computerized technologies either in form of wearable devices or an electronic health record database has helped improve the management of some chronic diseases through the integration of geospatial and clinical data [39, 40].

In cancer research, AI is widely used to evaluate the degree of tumor invasion, predict the course of the disease and prognosis, and give the advice on treatment, especially provides a strong analytical support for the study of breast cancer [41], hepatocellular carcinoma [42], and nasopharyngeal carcinoma [43]. Recently, the US Food and Drug Administration (FDA) has licensed several AI systems to develop testing devices for early diagnosis of cancer [44].

Through the AI algorithm, dozens or even hundreds of groups of data can be analyzed in detail to reveal the inherent laws of diseases and find out the associated factors of the occurrence, development, treatment, and prognosis of some chronic diseases, which is beyond the ability of human beings themselves. A prospective cohort study of 500,000 subjects was completed in the UK. Each subject provided data including biological measurements, lifestyle indicators, biomarkers in blood and urine, and brain imaging information. The researchers also collected genome-wide gene data of all subjects, aiming to look for genetic associations associated with chronic diseases and their characteristics by using big data analysis [45]. Also, in an eight-year study, 109 subjects at high risk of type 2 diabetes received each quarter a measuring and sampling, including the clinical signs measurement, group analysis (genome, immunome, transcriptome, proteome, metabolome, and microbiome), and wearable equipment measurement. Finally, the analysis revealed 67 clinically actionable health discoveries and developed a predictive model for insulin resistance [46]. Without AI-based machine learning algorithms, such huge and complex data analysis was unimaginable in the past. Machine learning is also widely used in the field of nutrition to develop personalized diet management and prevent diet-related diseases [47].

\section{Application Prospect of AI in Rehabilitation and Management of MG}

4.1. Establish Monitoring System of Medication Safety and Adverse Events. Long-term treatment with immunosuppressive drugs is essential for most MG patients, and glucocorticoids in particular are irreplaceable. Oral prednisone is recognized as the first-line immunotherapy for MG, but the cumulative exposure dose of prednisone is associated with an increased risk of adverse reactions, including obesity, osteoporosis, abnormal glucose metabolism, and infection. Nonsteroidal immunosuppressants (such as azathioprine, mycophenolate mofetil, cyclosporine, tacrolimus, cyclophosphamide, and methotrexate) are recommended to be used in combination with glucocorticoids or alone for long-term (even lifelong) treatment $[10,11]$. Treatment-related adverse event (AE) arising due to immunosuppressive therapy in the initial months should be strictly monitored, such as leukopenia, thrombocytopenia, hepatic dysfunction (particularly with azathioprine treatment), or renal dysfunction (more common with cyclosporine treatment) [48], which has often been neglected in the past. According to statistics, over $90 \%$ of $\mathrm{AE}$ or serious adverse event (SAE) are not reported in spontaneous reporting systems [49]. Through the digital management platform, an effective utilization of AI technology could send reminders of drug safety monitoring to patients regularly, process the acquired monitoring data in real time, warn patients and doctors of abnormal data, and 
realize remote monitoring of treatment. Remote management of MG may work to be of much use for the aged patients with travelling difficulties, or in geographically challenged territories, or in times when healthcare facilities are not easily accessible such as pandemic periods.

4.2. Conduct Personal Life Guidance and Disease-Related Education. Patients using steroids should be supplemented with calcium and vitamin $\mathrm{D}$ and should be given with bisphosphonate therapy appropriately. At the same time, a generally healthy diet should be advised for all patients. It is worth noting that methotrexate and cyclophosphamide should be avoided in women of childbearing age because of their teratogenic effects. Some drugs associated with MG deterioration need to be used with caution and only upon suitable prescriptions from the doctors [48]. The similar notes and related knowledge, including diet, lifestyle, sleep, stress, and exercise habits, should be easily accessible to MG patients at any time on the management platform, and relevant safety warnings should be made according to the data generated by the patients. The management platform should be set up with an education section and constantly updated to improve the patient's self-management skills. It is worth learning from the application of $\mathrm{AI}$ in the individualized management of diabetes patients, including AI assisting diabetic patients to make scientific diet plan, carry out appropriate physical exercise, monitor the occurrence and development of common complications, and even provide technical support for patients in blood glucose monitoring and insulin use $[21,32]$.

\subsection{Develop a Social Platform for Mutual Benefit between} Doctors and Patients. The digital management platform itself also has the social function of communication. The growing partnership and cooperation are the foundation of development. Doctors can recruit the clinical trial subjects, spread knowledge, and demonstrate professionalism through the platform; patients can gain knowledge; understand condition; develop their own social network; read news; and share blogs, photos, and videos.

4.4. Participate in the Research Data of MG Rehabilitation and Management. Although, as mentioned above, clinical research on MG rehabilitation is currently facing difficulties, and there seems to be an opportunity to break through this bottleneck with the continuous maturity of AI technology and the development of information and communication technologies. First, AI will be applied to subject recruitment. After the establishment of MG management platform, patient information will be stored and accumulated and, gradually, developed into a valuable clinical resource database. When a study needs to recruit subjects, AI can preliminarily screen and match qualified subjects according to the inclusion and exclusion criteria and, then, recommend candidate subjects to doctors and provide their contact information so as to improve the efficiency of recruitment, expand recruitment coverage, and influence and achieve the best match. Instead of making regular trips to the hospital to participate in clinical trials, patients will receive remote monitor- ing, treatment, and life guidance. This AI-based clinical trial matching system has been successfully tested at the Mayo Clinic [50]. AI will provide more benefits to the subjects and improve their compliance. For example, AI can provide doctors with automatic, continuous, and real-time monitoring information from subjects through the management platform, so that subjects can get more attention from doctors.

In addition, wearable sensors and video surveillance can be used to automatically and continuously collect patient data, which is becoming increasingly available through the introduction of App-based and sensor-based exercise and therapy management systems [51]. For example, a trial of telerehabilitation after stroke [52] and a study in Parkinson's disease [53], both used data from sensors that measured body movement. For MG, we propose to establish a digital management platform that allows patients in consultation with physiotherapists to allocate patient-specific physical regimen to either the patient or the caregiver. The mobile technology may also allow the collection of adherence data. This realtime collection of data enables to minimized loss of data and ensures volume, variety, and velocity of data collection. The three V's of big data era will allow trend detection and correlations through the application of big data analytic tools. In the future, when enough available data are obtained, big data technologies will use all types of full data (not sample data) to draw reliable statistical conclusions, instead of extrapolating the real world around a small sample of data.

\section{Conclusion}

MG is an incurable disease. Rehabilitation and individualized patient management are essential components of MG lifetime therapy. In the future, drawing on the successful experience of AI in other chronic disease management, it is an unstoppable trend to develop MG digital management platform with functions such as education, management, social contact, and access to research data.

\section{Abbreviations}

AE: adverse event

AI: artificial intelligence

CSR: complete stable response

EHR: electronic health records

FDA: Food and Drug Administration

IVIg: intravenous immunoglobulin

MG: myasthenia gravis

MGFA: Myasthenia Gravis Foundation of America

MMS: minimum performance state

NCDs: noncommunicable diseases

SAE: serious adverse event.

\section{Data Availability}

This article has no additional data.

\section{Conflicts of Interest}

The authors declare that they have no conflicts of interests. 


\section{Acknowledgments}

This work was supported by the research fund of the Second Affiliated Hospital of Harbin Medical University (KYBS2015-14 and KYCX2018-18) and the Heilongjiang postdoctoral fund (LBH-Z16149).

\section{References}

[1] N. E. Gilhus, "Myasthenia gravis," The New England Journal of Medicine, vol. 375, no. 26, pp. 2570-2581, 2016.

[2] N. E. Gilhus and J. J. Verschuuren, "Myasthenia gravis: subgroup classification and therapeutic strategies," Lancet Neurology, vol. 14, no. 10, pp. 1023-1036, 2015.

[3] A. S. Carr, C. R. Cardwell, P. O. McCarron, and J. McConville, "A systematic review of population based epidemiological studies in Myasthenia Gravis," BMC Neurology, vol. 10, no. 1, p. $46,2010$.

[4] J. F. Owe, A. K. Daltveit, and N. E. Gilhus, "Causes of death among patients with myasthenia gravis in Norway between 1951 and 2001," Journal of Neurology, Neurosurgery, and Psychiatry, vol. 77, no. 2, pp. 203-207, 2006.

[5] J. S. Hansen, D. H. Danielsen, F. E. Somnier et al., "Mortality in myasthenia gravis: a nationwide population-based follow-up study in Denmark," Muscle \& Nerve, vol. 53, no. 1, pp. 7377, 2016.

[6] D. Grob, N. Brunner, T. Namba, and M. Pagala, "Lifetime course of myasthenia gravis," Muscle \& Nerve, vol. 37, no. 2, pp. 141-149, 2008.

[7] L. Padua, A. Evoli, I. Aprile et al., "Health-related quality of life in patients with myasthenia gravis and the relationship between patient-oriented assessment and conventional measurements," Neurological Sciences, vol. 22, no. 5, pp. 363-369, 2001.

[8] R. H. Paul, J. M. Nash, R. A. Cohen, J. M. Gilchrist, and J. M. Goldstein, "Quality of life and well-being of patients with myasthenia gravis," Muscle \& Nerve, vol. 24, no. 4, pp. 512516, 2001.

[9] S. Twork, S. Wiesmeth, J. Klewer, D. Pöhlau, and J. Kugler, "Quality of life and life circumstances in German myasthenia gravis patients," Health and Quality of Life Outcomes, vol. 8, no. 1, p. 129, 2010.

[10] D. B. Sanders, G. I. Wolfe, M. Benatar et al., "International consensus guidance for management of myasthenia gravis: executive summary," Neurology, vol. 87, no. 4, pp. 419-425, 2016.

[11] P. Narayanaswami, D. B. Sanders, G. Wolfe et al., "International consensus guidance for management of myasthenia gravis: 2020 update," Neurology, vol. 96, no. 3, pp. 114-122, 2021.

[12] T. M. Burns, C. K. Grouse, M. R. Conaway, D. B. Sanders, and MG Composite and MG-QOL15 Study Group, "Construct and concurrent validation of the MG-QOL15 in the practice setting," Muscle \& Nerve, vol. 41, no. 2, pp. 219-226, 2010.

[13] T. M. Burns, C. K. Grouse, G. I. Wolfe, M. R. Conaway, D. B. Sanders, and MG Composite and MG-OL15 Study Group, "The MG-QOL15 for following the health-related quality of life of patients with myasthenia gravis," Muscle \& Nerve, vol. 43, no. 1, pp. 14-18, 2011.

[14] T. M. Burns, R. Sadjadi, K. Utsugisawa et al., "International clinimetric evaluation of the MG-QOL15, resulting in slight revision and subsequent validation of the MG-QOL15r," Muscle \& Nerve, vol. 54, no. 6, pp. 1015-1022, 2016.

[15] H. Murai, "Japanese clinical guidelines for myasthenia gravis: putting into practice," Clinical and Experimental Neuroimmunology, vol. 6, no. 1, pp. 21-31, 2015.

[16] H. H. Kyu, V. F. Bachman, L. T. Alexander et al., "Physical activity and risk of breast cancer, colon cancer, diabetes, ischemic heart disease, and ischemic stroke events: systematic review and dose-response meta-analysis for the Global Burden of Disease Study 2013,” BMJ, vol. 354, 2016.

[17] R. J. Thomas, S. A. Kenfield, and A. Jimenez, "Exerciseinduced biochemical changes and their potential influence on cancer: a scientific review," British Journal of Sports Medicine, vol. 51, no. 8, pp. 640-644, 2017.

[18] P. Kokkinos, "Physical activity, health benefits, and mortality risk,” ISRN Cardiology, vol. 2012, Article ID 718789, 2012.

[19] M. A. Rahbek, E. E. Mikkelsen, K. Overgaard, L. Vinge, H. Andersen, and U. Dalgas, "Exercise in myasthenia gravis: a feasibility study of aerobic and resistance training," Muscle \& Nerve, vol. 56, no. 4, pp. 700-709, 2017.

[20] E. Westerberg, C. J. Molin, I. Lindblad, M. Emtner, and A. R. Punga, "Physical exercise in myasthenia gravis is safe and improves neuromuscular parameters and physical performance-based measures: a pilot study," Muscle \& Nerve, vol. 56, no. 2, pp. 207-214, 2017.

[21] G. A. Fregonezi, V. R. Resqueti, R. Güell, J. Pradas, and P. Casan, "Effects of 8-week, interval-based inspiratory muscle training and breathing retraining in patients with generalized myasthenia gravis," Chest, vol. 128, no. 3, pp. 1524-1530, 2005.

[22] I. Contreras and J. Vehi, "Artificial intelligence for diabetes management and decision support: literature review," Journal of Medical Internet Research, vol. 20, no. 5, article e10775, 2018.

[23] S. M. McKinney, M. Sieniek, V. Godbole et al., "International evaluation of an AI system for breast cancer screening," Nature, vol. 577, no. 7788, pp. 89-94, 2020.

[24] M. D. Abràmoff, Y. Lou, A. Erginay et al., "Improved automated detection of diabetic retinopathy on a publicly available dataset through integration of deep learning," Investigative Ophthalmology \& Visual Science, vol. 57, no. 13, pp. 52005206, 2016.

[25] J. de Fauw, J. R. Ledsam, B. Romera-Paredes et al., "Clinically applicable deep learning for diagnosis and referral in retinal disease," Nature Medicine, vol. 24, no. 9, pp. 1342-1350, 2018.

[26] A. Esteva, B. Kuprel, R. A. Novoa et al., "Dermatologist-level classification of skin cancer with deep neural networks," Nature, vol. 542, no. 7639, pp. 115-118, 2017.

[27] P. Rajpurkar, J. Irvin, R. L. Ball et al., "Deep learning for chest radiograph diagnosis: a retrospective comparison of the CheXNeXt algorithm to practicing radiologists," PLoS Medicine, vol. 15, no. 11, article e1002686, 2018.

[28] L. M. Fleuren, T. L. T. Klausch, C. L. Zwager et al., "Machine learning for the prediction of sepsis: a systematic review and meta-analysis of diagnostic test accuracy," Intensive Care Medicine, vol. 46, no. 3, pp. 383-400, 2020.

[29] J. Yim, R. Chopra, T. Spitz et al., "Predicting conversion to wet age-related macular degeneration using deep learning," Nature Medicine, vol. 26, no. 6, pp. 892-899, 2020.

[30] H. Kim, J. M. Goo, K. H. Lee, Y. T. Kim, and C. M. Park, "Preoperative CT-based deep learning model for predicting 
disease-free survival in patients with lung adenocarcinomas," Radiology, vol. 296, no. 1, pp. 216-224, 2020.

[31] P. Wang, T. M. Berzin, J. R. Glissen Brown et al., "Real-time automatic detection system increases colonoscopic polyp and adenoma detection rates: a prospective randomised controlled study," Gut, vol. 68, no. 10, pp. 1813-1819, 2019.

[32] N. S. Tyler, C. M. Mosquera-Lopez, L. M. Wilson et al., "An artificial intelligence decision support system for the management of type 1 diabetes," Nature Metabolism, vol. 2, no. 7, pp. 612-619, 2020.

[33] J. Li, J. Huang, L. Zheng, and X. Li, “Application of artificial intelligence in diabetes education and management: present status and promising prospect," Frontiers in Public Health, vol. 8, p. 173, 2020.

[34] M. Subramanian, A. Wojtusciszyn, L. Favre et al., "Precision medicine in the era of artificial intelligence: implications in chronic disease management," Journal of Translational Medicine, vol. 18, no. 1, p. 472, 2020.

[35] GBD 2017 Disease and Injury Incidence and Prevalence Collaborators, "Global, regional, and national incidence, prevalence, and years lived with disability for 354 diseases and injuries for 195 countries and territories, 1990-2017: a systematic analysis for the Global Burden of Disease Study 2017," Lancet, vol. 392, no. 10159, pp. 1789-1858, 2018.

[36] G. Fico, M. T. Arredondo, V. Protopappas, E. Georgia, and D. Fotiadis, "Mining data when technology is applied to support patients and professional on the control of chronic diseases: the experience of the METABO platform for diabetes management," Methods in Molecular Biology, vol. 1246, pp. 191-216, 2015.

[37] G. Fico, A. Fioravanti, M. T. Arredondo et al., "Integration of personalized healthcare pathways in an ICT platform for diabetes managements: a small-scale exploratory study," IEEE Journal of Biomedical and Health Informatics, vol. 20, no. 1, pp. 29-38, 2016.

[38] P. Porter, U. Abeyratne, V. Swarnkar et al., "A prospective multicentre study testing the diagnostic accuracy of an automated cough sound centred analytic system for the identification of common respiratory disorders in children," Respiratory Research, vol. 20, no. 1, p. 81, 2019.

[39] M. V. McConnell, A. Shcherbina, A. Pavlovic et al., "Feasibility of obtaining measures of lifestyle from a smartphone app: the MyHeart Counts Cardiovascular Health Study," JAMA Cardiology, vol. 2, no. 1, pp. 67-76, 2017.

[40] T. Althoff, R. Sosič, J. L. Hicks, A. C. King, S. L. Delp, and J. Leskovec, "Large-scale physical activity data reveal worldwide activity inequality," Nature, vol. 547, no. 7663, pp. 336339, 2017.

[41] Q. Li, W. Li, J. Zhang, and Z. Xu, “An improved k-nearest neighbour method to diagnose breast cancer," The Analyst, vol. 143, no. 12, pp. 2807-2811, 2018.

[42] G. Qiao, J. Li, A. Huang, Z. Yan, W. Y. Lau, and F. Shen, “Artificial neural networking model for the prediction of posthepatectomy survival of patients with early hepatocellular carcinoma," Journal of Gastroenterology and Hepatology, vol. 29, no. 12, pp. 2014-2020, 2014.

[43] W. Zhu and X. Kan, "Neural network cascade optimizes microRNA biomarker selection for nasopharyngeal cancer prognosis," PLoS One, vol. 9, no. 10, article e110537, 2014.

[44] S. Gerke, B. Babic, T. Evgeniou, and I. G. Cohen, "The need for a system view to regulate artificial intelligence/machine learning-based software as medical device," NPJ Digital Medicine, vol. 3, no. 1, p. 53, 2020.

[45] C. Bycroft, C. Freeman, D. Petkova et al., "The UK Biobank resource with deep phenotyping and genomic data," Nature, vol. 562, no. 7726, pp. 203-209, 2018.

[46] S. M. Schüssler-Fiorenza Rose, K. Contrepois, K. J. Moneghetti et al., "A longitudinal big data approach for precision health," Nat Med, vol. 25, no. 5, pp. 792-804, 2019.

[47] D. D. Wang and F. B. Hu, "Precision nutrition for prevention and management of type 2 diabetes," The Lancet Diabetes and Endocrinology, vol. 6, no. 5, pp. 416-426, 2018.

[48] N. E. Gilhus, S. Tzartos, A. Evoli, J. Palace, T. M. Burns, and J. J. G. M. Verschuuren, "Myasthenia gravis," Nature Reviews Disease Primers, vol. 5, no. 1, p. 30, 2019.

[49] L. Hazell and S. A. Shakir, "Under-reporting of adverse drug reactions: a systematic review," Drug Safety, vol. 29, no. 5, pp. 385-396, 2006.

[50] J. Helgeson, M. Rammage, A. Urman et al., "Clinical performance pilot using cognitive computing for clinical trial matching at Mayo Clinic," Journal of Clinical Oncology, vol. 36, 15 suppl, p. e18598, 2018.

[51] Q. Wang, P. Markopoulos, B. Yu, W. Chen, and A. Timmermans, "Interactive wearable systems for upper body rehabilitation: a systematic review," Journal of Neuroengineering and Rehabilitation, vol. 14, no. 1, p. 20, 2017.

[52] L. Dodakian, A. L. McKenzie, V. le et al., "A home-based telerehabilitation program for patients with stroke," Neurorehabil Neural Repair, vol. 31, no. 10-11, pp. 923-933, 2017.

[53] C. S. Tucker, I. Behoora, H. B. Nembhard, M. Lewis, N. W. Sterling, and X. Huang, "Machine learning classification of medication adherence in patients with movement disorders using non-wearable sensors," Computers in Biology and Medicine, vol. 66, pp. 120-134, 2015. 